\title{
Acute Phase Treatment and Medium-Term Outcomes in Kawasaki Disease
}

\author{
Toshimasa Nakada
}

\begin{abstract}
An acute phase treatment for prevention of coronary artery stenosis caused by Kawasaki disease (KD) has not been established. The objective of this study was to clarify the medium-term outcomes of patients who received acute phase treatment in our department. This retrospective study included data from 214 patients with KD who received acute phase treatment from January 2009 to May 2020. A total of 196 (92.1\%) received an initial single dose of intravenous immunoglobulin (IVIG) therapy. One patient with status epilepticus at presentation received initial IVIG plus steroid therapy. A total of 17 patients did not receive IVIG. The rate of coronary artery lesions (CALs) 1 month and 1 year after KD onset were $1.9 \%$ and $0.9 \%$, respectively. Two patients had CAL 1 year after KD onset. However, no patients had coronary artery stenosis. One patient with a right giant CAL had a medium CAL before initial therapy. During a median follow-up period of 3 years and 4 months, no patients had cardiac events that required therapy.
\end{abstract}

Index Terms - Coronary artery lesions, Intravenous immunoglobulin therapy, Kawasaki disease, Outcomes.

\section{INTRODUCTION}

Kawasaki disease (KD) is an acute systemic vasculitis of unknown cause that mostly affects infants and children [1]. Coronary artery lesions (CALs) are a severe complication of KD. However, an acute phase treatment to prevent coronary artery stenosis has not been established. Studies regarding acute phase treatment for KD have been published [2]-[5]. However, a limitation is that their primary endpoints were the outcomes of CALs 6-8 weeks after KD onset, and the value of long-term outcomes of CALs require follow-up [6].

The standard therapy for the acute KD phase includes intravenous immunoglobulin (IVIG) therapy at $2 \mathrm{~g} / \mathrm{kg} / \mathrm{dose}$ with concomitant medium-dose or high-dose aspirin [7]. However, the addition of medium-dose or high-dose aspirin is now controversial [8]. A randomized controlled trial on the effectiveness of IVIG monotherapy and IVIG combined with high-dose aspirin in the acute $\mathrm{KD}$ stage is ongoing [9]. Studies suggest that aspirin may inhibit CAL prevention and that delayed use of aspirin (DUA) may be beneficial for the prevention of coronary artery stenosis in KD [10]-[13]. This study aimed to clarify the medium-term outcomes of patients who received acute phase treatment in our department and ascertain the usefulness of an initial single dose of IVIG therapy $(2 \mathrm{~g} / \mathrm{kg} / \mathrm{dose})$ with DUA to prevent coronary artery stenosis in patients without CALs before initial treatment.

Published on August 11, 2020.

Toshimasa Nakada, Aomori Prefectural Central Hospital, Japan.

(corresponding e-mail: toshimasanakada@yahoo.co.jp)

\section{PARTICIPANTS AND METHODS}

Our institutional ethics committee approved the study protocol and waived the requirement of patient consent due to the retrospective nature of the study.

This retrospective study included data from 214 patients who received acute phase treatment for KD between January 2009 and May 2020. KD diagnoses were established based on the criteria (Japanese, 5th edition) mentioned in the diagnostic guidelines for KD until August 2019 and based on the revised criteria (Japanese, 6th edition) from September 2019 [14], [15]. Patients with a first episode of KD were included. One patient with heart failure at presentation who was transferred to another hospital was excluded because his detailed clinical data were not available. The remaining patients were divided into three groups: those with complications at presentation who received IVIG therapy (pre-complication group, $\mathrm{n}=5$ ), those without complications at presentation who received IVIG therapy with DUA (DUA group, $\mathrm{n}=192)$, and those who received acute phase treatment without IVIG therapy (non-IVIG group, $\mathrm{n}=17$ ). The outcomes were the rates of CALs and coronary artery stenosis 1 year after KD onset.

IVIG resistance was defined as fever that persisted or reappeared $24 \mathrm{~h}$ after initial treatment [16]. KD presentation was defined as relapse when a second episode occurred within 2 months of the first episode after initial defervescence [17], [18]. Defervescence was defined as a body temperature $<37.5^{\circ} \mathrm{C}$ for at least $24 \mathrm{~h}$, and the defervescence time was defined as the moment when the body temperature reached < $37.5{ }^{\circ} \mathrm{C}$. Recurrent $\mathrm{KD}$ was defined as a secondary presentation of another episode 2 months after an initial episode [17], [18].

\section{A. Initial Therapy}

During the study period, an initial single IVIG infusion of $2 \mathrm{~g} / \mathrm{kg} / \mathrm{dose}$, starting on day 5 of the illness, was used as initial therapy, whenever possible for the DUA group (Fig. 1). Prompt initial treatment was started for patients in the precomplication group at presentation. If the patients had CALs, IVIG therapy with concomitant aspirin was started. Patients without inflammation or complications at presentation received acute phase therapy without IVIG.

Between January 2009 and November 2017, antiinflammatory drugs (aspirin or flurbiprofen) were initiated within $24 \mathrm{~h}$ after the end of the initial IVIG infusion for patients in the DUA group (Fig. 1) [10]. Aspirin was initiated at a dose of $30 \mathrm{mg} / \mathrm{kg} /$ day and decreased to $5-10 \mathrm{mg} / \mathrm{kg} /$ day 
once the patient was afebrile. Flurbiprofen was initiated at a dose of $3-5 \mathrm{mg} / \mathrm{kg} /$ day and decreased to $3 \mathrm{mg} / \mathrm{kg} /$ day once the patient was afebrile [10]. Each treating physician chose between aspirin and flurbiprofen after considering the patient's liver function and the risk of Reye syndrome during the influenza season. After December 2017, low-dose aspirin $(5 \mathrm{mg} / \mathrm{kg} /$ day) was initiated on day 8 to 10 of illness after completion of the IVIG infusion, including the second course of therapy [18].

\section{Day 5 Initial single IVIG therapy ( $2 \mathrm{~g} / \mathrm{kg} / \mathrm{dose})$ \\ Day $8 \quad 2^{\text {nd }}$ IVIG therapy $(2 \mathrm{~g} / \mathrm{kg} / \mathrm{dose})$ \\ Day $9 \quad 3^{\text {rd }}$ course therapy \\ (plasma exchange, $3^{\text {rd }}$ IVIG therapy, or ulinastatin)

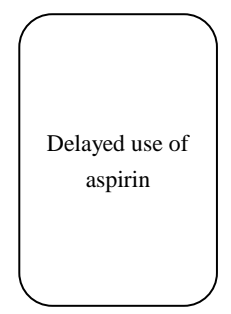

Fig. 1. Acute phase treatment for the DUA group.

IVIG: intravenous immunoglobulin, DUA: delayed use of aspirin.

\section{B. Rescue Therapy}

The decision to use rescue therapies in resistant patients was made between 48 and $72 \mathrm{~h}$ after the initial IVIG therapy was completed (Fig. 1). The decision was comprehensively made according to clinical parameters, including body temperature, major KD symptoms, general condition, and laboratory data [19]. The second course of therapy was rescue IVIG therapy at $2 \mathrm{~g} / \mathrm{kg} / \mathrm{dose}$, and the third course of therapy was a ulinastatin infusion, a third course of IVIG therapy, or plasma exchange (Fig. 1) [13],[18].

\section{Diagnosis of CAL}

CALs were diagnosed using echocardiography based on the Japanese criteria according to Kobayashi et al [20]. A CAL was diagnosed if any of the examinations showed an internal lumen diameter $\geq 3 \mathrm{~mm}$ in a patient younger than 5 years or a diameter $\geq 4 \mathrm{~mm}$ in a patient older than 5 years, if the internal diameter of a segment was at least 1.5 times that of an adjacent segment, or if the lumen appeared irregular. A transient CAL was defined as the disappearance of a CAL within 30 days of the illness.

\section{Statistical Analysis}

Statistical analyses were performed using Stat Flex Version 6 for Windows (Artech Co., Ltd., Osaka, Japan). Chisquare, Fisher's exact, and Mann-Whitney U tests were used as appropriate, with sample size considerations. A P value of $<0.05$ was considered statistically significant.

\section{RESULTS}

Of the 214 patients, $4(1.9 \%)$ had a CAL before initial treatment (Table I). No patients in the DUA group had abnormal findings on the first echocardiogram. A total of 197 patients $(92.1 \%)$ received IVIG therapy on a median 5th day of illness (Tables I and II). Of the 197 patients, 193 (98.0\%) received an initial $2 \mathrm{~g} / \mathrm{kg} / \mathrm{dose}$ of IVIG therapy (Table II), and $27(13.7 \%)$ received rescue therapy after initial therapy. Of the 197 patients, $5(2.5 \%)$ received third course of therapy, and 2 patients received plasma exchange (Table II). Of the 214 patients, $6(2.8 \%)$ experienced KD relapse. However, no patients with relapse had a CAL. No patients experienced significant adverse effects due to acute phase treatment, such as shock, hemolytic anemia, and complications of severe infections (e.g., sepsis or osteomyelitis).

TABLE I: DEMOGRAPHIC DATA OF 214 PATIENTS

\begin{tabular}{|l|l|}
\hline Variables & Data \\
\hline Gender & Male/female = 109/105 \\
\hline Age at KD onset & $\begin{array}{l}\text { Median 2 years and 2.5 months (range: 2 months- } \\
13 \text { years and 3 months) }\end{array}$ \\
\hline Incomplete type & $41(19.2 \%)$ \\
\hline Patients with complications & $5(2.3 \%)$ \\
before initial treatment & CAL \\
\hline IVIG therapy & Status epilepticus 1 (0.5\%) \\
\hline Low-dose aspirin/medium-dose & $41(19.2 \%) / 106(49.5 \%) / 67(31.3 \%)$ \\
\hline aspirin/flurbiprofen & \\
\hline CAL & $9(4.2 \%)$ \\
\hline Less than one month after KD & $9(4.2 \%)$ \\
\hline onset & $4(1.9 \%)$ \\
\hline One month after KD onset & $2(0.9 \%)$ \\
\hline One year after KD onset & \\
\hline
\end{tabular}

Data are present4ed as n (\%). KD: Kawasaki disease.

Incomplete type: major signs of $\mathrm{KD} \leq 4$, IVIG: intravenous immunoglobulin, CAL: coronary artery lesion.

The rates of CALs less than one month and 1 month after KD onset were $4.2 \%$ and $1.9 \%$, respectively, in the 214 patients (Table I). The rates of CALs in the pre-complication, DUA, and non-IVIG group patients less than 1 month after KD onset were $80.0 \%, 2.7 \%$, and $0.0 \%$, respectively, and those rates significantly differed among the three groups $(\mathrm{P}<$ 0.01). The rate of concomitant use of aspirin and initial IVIG therapy in the pre-complication group patients was significantly higher than that in the DUA group patients (Table III). The protocol of the initial IVIG therapies significantly differed between the pre-complication and the DUA group patients (Table III). The rates of CALs less than 1 month and 1 month after KD onset in the pre-complication group patients were significantly higher than those in the DUA group patients (Table III). Of the 214 patients, 2 (0.9\%) had a CAL 1 year after KD onset (Table I ), and their selective coronary angiograms showed findings with right CALs and normal left coronary arteries. No patients had coronary artery stenosis. Although not statistically significant, the rate of CALs in the pre-complication group patients 1 year after KD onset was higher than that in the DUA group patients (Table III).

The median follow-up period of the 214 patients was 3 years and 4 months (range: 13 days -9 years and 8 months). Among the 214 patients, 93 patients $(43.5 \%)$ were still undergoing follow-up, and 80 patients (37.4\%) had 
completed follow-up at the last visit. A total of 21 patients $(9.8 \%)$ were transferred to another hospital during follow-up, and 20 patients $(9.3 \%)$ were lost to follow-up. Four patients with CALs 1 month after KD onset had ongoing follow-up at the last visit. During follow-up, 5 of the 214 patients (2.3\%) experienced KD recurrence. However, no patients with recurrence had CALs. Two patients had chest pain syndrome, and one patient had mild aortic valve regurgitation 6 years after KD onset. However, no patients had cardiac events that required therapy, such as myocardial infarction/ischemia, heart failure, or life-threatening arrhythmia.

TABLE II: TREATMENT AND OUTCOMES OF 197 PATIENTS WHO RECEIVED IVIG THERAPY

\begin{tabular}{|c|c|}
\hline Variables & Data \\
\hline $\begin{array}{l}\text { Day of illness at initial IVIG } \\
\text { therapy }\end{array}$ & Median 5 (range: 3-16) \\
\hline Protocol of initial IVIG therapy & $\begin{array}{l}2 \mathrm{~g} / \mathrm{kg} / \text { dose: } 193(98.0 \%) \\
2 \mathrm{~g} / \mathrm{kg} / \text { dose }+ \text { steroid: } 1(0.5 \%) \\
1 \mathrm{~g} / \mathrm{kg} / \text { dose for } 2 \text { days: } 3(1.4 \%)\end{array}$ \\
\hline Non-responder & $43(21.8 \%)$ \\
\hline Rescue therapy & $\begin{array}{cc}2^{\text {nd }} \text { IVIG therapy: } & 27(13.7 \%) \\
\text { For non-responder: } & 20(10.2 \%) \\
\text { For relapser: } & 6(3.0 \%) \\
\text { For responder: } & 1(0.5 \%) \\
3^{\text {rd }} \text { course therapy: } & 5(2.5 \%) \\
\text { Plasma exchange: } & 2(1.0 \%) \\
3^{\text {rd }} \text { IVIG therapy: } & 2(1.0 \%) \\
\text { Ulinastatin: } & 1(0.5 \%)\end{array}$ \\
\hline
\end{tabular}

Data are presented as n (\%). KD: Kawasaki disease.

IVIG: intravenous immunoglobulin, CAL: coronary artery lesion.

\section{DISCUSSION}

The main findings of this study were that the medium-term outcomes of patients with $\mathrm{KD}$ who received acute phase treatment in our department were favorable and that the rate of CALs and coronary artery stenosis 1 year after onset were $0.9 \%$ and $0.0 \%$, respectively. Moreover, an initial single dose of IVIG therapy ( $2 \mathrm{~g} / \mathrm{kg} / \mathrm{dose})$ with DUA for patients without complications before initial treatment was useful for the prevention of coronary artery stenosis.

An initial single dose of IVIG therapy ( $2 \mathrm{~g} / \mathrm{kg} / \mathrm{dose})$ with DUA has benefits regarding prevention of coronary artery stenosis, low rates of KD relapse, cost-effectiveness, and protection against severe infections.

The major goal of acute phase $\mathrm{KD}$ treatment is the prevention of coronary artery stenosis that may lead to myocardial ischemia [21]. During the median follow-up period of 3 years and 4 months, no patients had cardiac events that required therapy, such as cardiac infarction and myocardial ischemia. CAL size is significantly related to the occurrence of myocardial infarction/ischemia [22]. Therefore, CAL size during the acute phase of $\mathrm{KD}$ is an important factor for late phase outcomes. The cut-off values for CALs within the first 100 days after KD onset leading to a stenotic lesion in the late phase of $\mathrm{KD}$, were a diameter $\geq$
$6.1 \mathrm{~mm}$ in patients with a body surface area $<0.50 \mathrm{~m}^{2}$ and a diameter $\geq 8.0 \mathrm{~mm}$ in patients with a body surface area $\geq 0.50$ $\mathrm{m}^{2}$ [23]. One study regarding initial IVIG therapy with DUA showed that the diameters of CALs in the DUA group patients were not larger than these cut-off values two months after KD onset [13]. Moreover, another study demonstrated an initial single dose of IVIG therapy with DUA was useful for prevention of CALs $\geq 3 \mathrm{~mm}$ in infants younger than 1 year of age who had a high risk of CAL during the acute phase of KD [24]. This cohort study confirmed the efficacy of initial single dose of IVIG therapy with DUA for prevention of coronary artery stenosis 1 year after KD onset.

TABLE III: COMPASION OF CLINICAL FINDINGS, TREATMENTS AND OUTCOMES BETWEEN THE PRE- COMPLICATION GROUP AND THE DUA GROUP

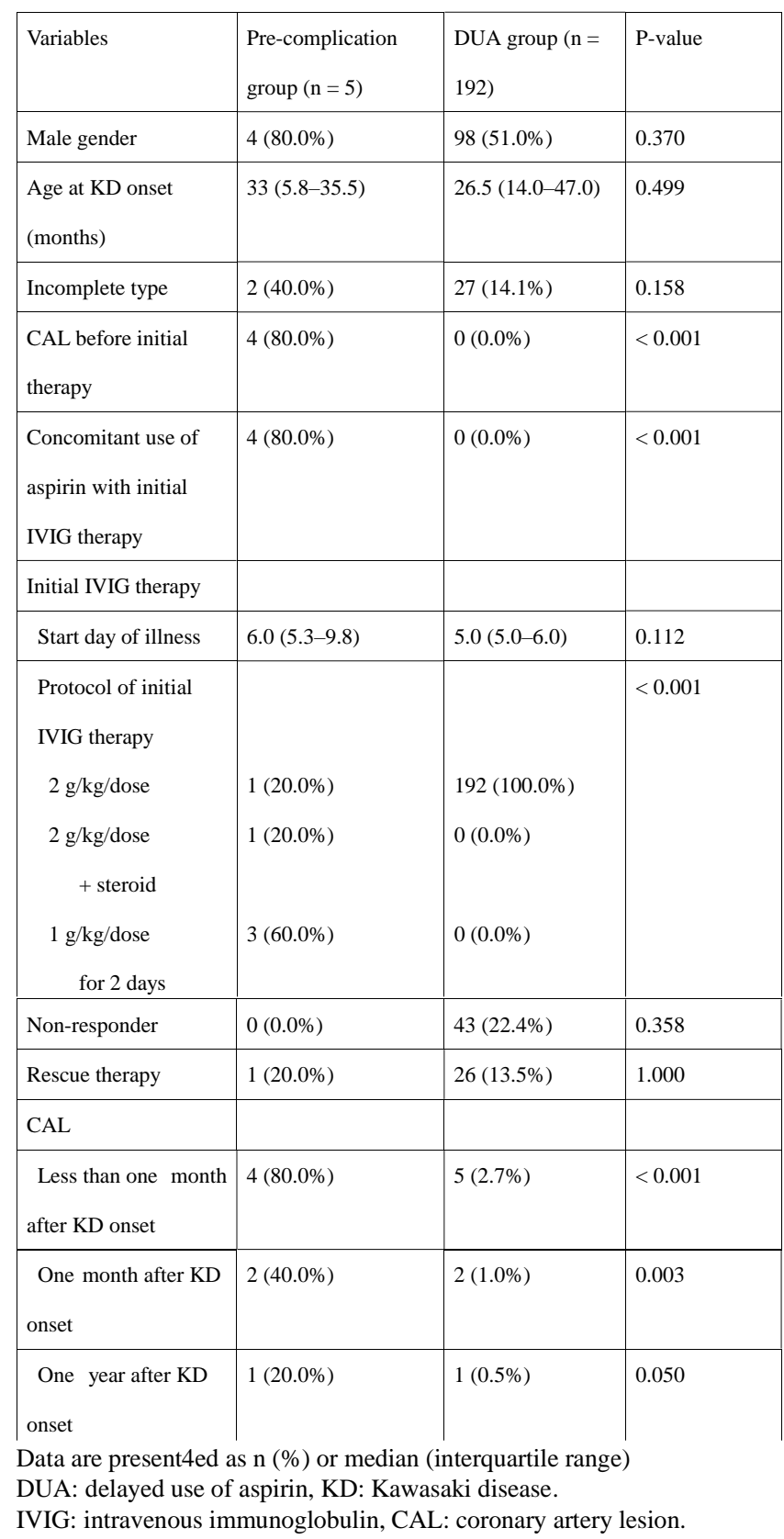

Appropriate use of IVIG therapy and plasma exchange was useful for prevention of coronary artery stenosis in this study. Two patients who received plasma exchange had no CALs 1 year after KD onset. Although one patient had a CAL with a maximal size of $4.8 \mathrm{~mm}$ on the right proximal coronary artery 
during the acute phase of $\mathrm{KD}$, the CAL regressed 2 months after KD onset [13]. Another patient had no CALs during either the acute or late phases of KD in this study. Takahara et al. found that rescue therapies, including plasma exchange before 10 days of illness, were useful for preventing large CALs in KD [25]. Moreover, rescue therapies using plasma exchange are considered useful for CAL suppression during the late KD stages [26]. Results regarding the patients who received plasma exchange in the present study were consistent with those of previous reports [25], [26].

Early identification of patients likely to develop IVIG resistance is a challenge [27] and identifying patients with initial IVIG resistance after initial therapy is easier. Several factors, including IVIG resistance, responsiveness, and disease relapse, are associated with CAL complications [28]. Therefore, risk stratification after initial therapy for KD is important for prevention of large CALs [19]. Single IVIG therapy does not modify the clinical course of $\mathrm{KD}$, and this characteristic allows clinicians to manage treatment progress and to provide rescue therapies for IVIG resistance and KD relapse at appropriate times. This point may be another factor for the favorable outcomes of the patients in this study.

A nationwide epidemiologic survey of KD in Japan (201516) demonstrated that the rate of the patients who received IVIG therapy was $93.5 \%$ [29]. This rate is similar to that in the present study $(92.1 \%$, Table I). The rate of CALs in the non-IVIG group patients was $0.0 \%$. This finding suggests appropriate risk stratification before initial therapy and decision for using IVIG therapy among the patients in this study.

An initial single dose of IVIG therapy with DUA has a benefit regarding low rates of KD relapses. The rate of patients who received rescue therapies for KD relapses was $3.0 \%$ in the present study (Table II). IVIG therapy resistance during the acute phase of KD has been implicated in CAL development [20]. Trials have been performed to decrease the rate of IVIG resistance using the addition of prednisolone (RAISE study), infliximab, and cyclosporin (KAICA study) [2], [3], [5]. These trials were performed using IVIG therapy with concomitant use of medium-dose or high-dose aspirin [2], [3], [5]. The RAISE study and the KAICA study showed a significant decrease in the rate of IVIG resistance in the study group compared to that in the control group [2], [5]. However, the relapse rates in these studies were 5.1-fold to 12.7-fold higher than those in the study using DUA [2], [5], [18], [30]. Moreover, the KD relapse rate in the study group in the KAICA study was significantly higher than that in the IVIG-resistant patients in the study using DUA $(\mathrm{P}<0.001)$ [5], [18], [30].

$\mathrm{KD}$ relapse is caused by persistent inflammation and arteritis in the acute phase of KD and is a risk factor for CAL development as well as IVIG resistance [28]. These findings suggest that intensified initial IVIG therapies using concomitant medium-dose aspirin and cyclosporin/steroids are not able to control persistent inflammation and arteritis after the initial therapy. Moreover, the rates of CAL development using Japanese criteria were similar among the study group and the control group in the KAICA study and the IVIG-resistant patients in the study using DUA [30].

An initial single IVIG therapy with DUA has a benefit regarding cost-effectiveness. A recent Japanese nationwide survey regarding KD patients in 2015 and 2016 showed that the rates of rescue therapy and cardiac sequalae 1 month after onset were $21.8 \%$ and $2.3 \%$, respectively [29]. In the present study, those rates were $13.7 \%$ and $1.9 \%$, respectively. Another study showed the cost-effectiveness of the initial IVIG therapy with DUA regarding rescue therapies for IVIGresistant patients [30]. In the RAISE study and the KAICA study, patients who were predicted to be resistant to IVIG were enrolled [2], [5]. The patients were those with risk scores of five points or higher using a risk scoring system developed by Kobayashi et al [20]. The proportion of the predicted patients was $29.8 \%$ to $27.6 \%$ of the acute phase KD population [20],[31]. The proportion of the IVIG-resistant patients who received rescue therapies was $10.1 \%(21 / 207)$ in the study that utilized DUA [18]. The rate of the patients who received rescue therapy for IVIG resistance, response, and KD relapse in the DUA group was $13.5 \%$ in this study (Table III). This finding suggests the cost-effectiveness of the initial single IVIG therapy with DUA for KD.

Safety against severe infections is one of the benefits of single dose IVIG therapy. In the present study, no patients had complications of severe infections. In contrast to steroids/cyclosporin A, a single dose IVIG therapy has no immunosuppressive effects. This point may be important in the era of coronavirus disease 2019 (COVID-19). Adverse effects of the acute phase treatment for KD using steroids/cyclosporin A include severe infections, such as osteomyelitis and bacteremia [31], [32]. Immunosuppressive agents, such as steroids and cyclosporin $\mathrm{A}$, have significant risk for complications of severe infections [31],[32]. A single IVIG therapy may be safer than the acute phase treatment using steroids/cyclosporin A against severe infections.

The rate of CAL in the pre-complication group patients was higher than that in the DUA group patients less than 1month and 1 month and 1 year after KD onset (Table III). Four of five pre-complication group patients had CALs before initial therapy, whereas no patient in DUA group had abnormal findings on the first echocardiogram (Table III). A study showed that all patients with persistent CALs 1 year after KD onset already had an abnormal initial echocardiogram and that the presence of any abnormalities on the initial echocardiogram may contribute to early identification of patients with severe disease [33]. Another study using multivariable analyses demonstrated that the lack of CAL regression and persistent large CAL in the late phase were associated with large coronary artery diameters at diagnosis [34]. The study using logistic regression analysis of our KD patients also showed that CAL before initial treatment is an independent risk factor for persistent CAL in late-stage KD [35]. Therefore, the higher risk for CAL in the pre-complication group patients before initial therapy may contribute a higher rate of CAL. Furthermore, a low rate of 2 $\mathrm{g} / \mathrm{kg} /$ dose initial single dose of IVIG therapy may also contribute higher rate of CAL (Table III).

One patient in the pre-complication group had a right giant CAL with a diameter $\geq 8 \mathrm{~mm} 1$ year after KD onset (Table III). Although this patient had no stenotic lesions at the time this study was conducted, right coronary occlusion/stenosis may develop in the future because giant CALs have a risk for these lesions [22]. However, the prevalence of asymptomatic complete occlusion is significantly higher in the right 
coronary artery, and a study showed a favorable outcome 5 years after KD onset in a patient with a right giant CAL [23], [36]. Most patients with giant CALs can lead ordinary lives with appropriate management [22]. Our patient with a right giant CAL had an uneventful course with aspirin and warfarin 1 year after KD onset.

Drug therapies have a limitation for the prevention of coronary artery stenosis caused by KD. The RAISE study showed the significantly lower rate of CAL in the study group 4 weeks after KD onset compared to that in the control group [2]. However, giant CALs were not prevented in the patients without CALs before initial therapy with this regimen [2]. A study showed that a patient who had received initial IVIG and prednisolone combination therapy developed a giant CAL after relapse [37]. Another study regarding intensified initial IVIG therapy showed the outcomes of CALs in terms of the size and regression rate 12 months after KD onset were similar among patients who received IVIG only, IVIG plus infliximab, or IVIG plus corticosteroids [38]. Furthermore, a study showed that the incidence and severity of CALs did not change notably after infliximab administration in Japanese patients with KD [39]. These findings suggest the limitation for prevention of coronary artery stenosis caused by KD among patients who received the mentioned pharmacotherapy regimens.

Rescue therapy with plasma exchange may be useful for prevention of coronary artery stenosis in high-risk patients based on the outcomes of the DUA group patients and the findings of previous studies [13], [25], [26]. Indication criteria for plasma exchange in patients with $\mathrm{KD}$ have not been established. However, biomarkers, such as the serum albumin level, C-reactive protein values, and neutrophil to lymphocyte ratio after initial therapy, may be useful for risk stratification after initial therapy [19], [40]-[42].

KD recurrence and development of arrhythmia are important problems after the acute phase of KD.

A nationwide epidemiologic survey of KD in Japan (201516) disclosed a $4.2 \%$ prevalence of KD recurrence [29]. The rate of $\mathrm{KD}$ recurrence was $2.3 \%$ in this cohort study. The incidence of KD recurrence is high among those with cardiac sequelae during the first episode [17]. The low rate of CALs during the first episode may be a factor for the lower rate of $\mathrm{KD}$ recurrence in this study. KD recurrence is a risk factor for CAL development [43]-[45]. In fact, 2 of 5 patients (40\%) with recurrence between 1991 and 2003 had medium-size CALs one month after the second episode in our department [45]. In the present cohort study, no patients with recurrence had CALs. The higher rate of patients who received 2 $\mathrm{g} / \mathrm{kg} / \mathrm{dose}$ IVIG therapy may be a factor for the different rates of CAL development compared with previous rates.

A study of 165 patients with a history of KD using exercise stress testing showed that ventricular arrhythmias were uncommon and were seen only in patients with medium or large CALs [46]. This finding was consistent with the findings of the present study. No patients, including the two patients with CALs one year after KD onset, had any significant arrhythmias in the late phase. A case report showed a patient who had ventricular arrhythmia and possible myocardial ischemia in late-stage KD despite no association with CALs with KD onset [47]. This patient was treated with aspirin monotherapy and did not receive IVIG therapy during the acute phase of KD [47]. Fortunately, the non-IVIG group patients in the present study had no significant arrhythmias or ischemic findings on exercise stress testing in the late phase. The complication of significant arrhythmias may be a rare event in patients without CALs in the late phase.

The limitations of this study include the small sample size and the retrospective study design.

In conclusion, the medium-term outcomes of patients with $\mathrm{KD}$ who received acute phase treatment in our department were favorable, and the rate of CALs and coronary artery stenosis one year after onset were $0.9 \%$ and $0.0 \%$, respectively. Moreover, initial single IVIG therapy (2 $\mathrm{g} / \mathrm{kg} / \mathrm{dose}$ ) with DUA for patients without CALs before initial treatment was useful for prevention of coronary artery stenosis. The majority of KD patients had favorable mediumterm outcomes with acute phase management using risk stratification before and after initial treatment, initial single IVIG therapy (2 $\mathrm{g} / \mathrm{kg} / \mathrm{dose})$ with DUA, and appropriate rescue therapies using IVIG and plasma exchange.

\section{ACKNOWLEDGMENT}

I would like to thank the pediatric cardiologists in Hirosaki University Hospital who provided clinical data regarding the patients who received plasma exchange and selective coronary angiography, all of those who were involved in the medical management of the patients included in this study, and Enago (www.enago.jp) for the English language review.

\section{REFERENCES}

[1] Burns JC, Glod é MP. Kawasaki syndrome. Lancet 2004; 364(9433): 533-44. https://doi.org/10.1016/S0140-6736(04)16814-1.

[2] Kobayashi T, Saji T, Otani T, Takeuchi K, Nakamura T, Arakawa H, et al. Efficacy of immunoglobulin plus prednisolone for prevention of coronary artery abnormalities in severe Kawasaki disease (RAISE study): a randomised, open-label, blinded-endpoints trial. Lancet. 2012; 379(9826):1613-20. https://doi.org/10.1016/S01406736(11)61930-2

[3] Tremoulet AH, Jain S, Jaggi P, Jimenez-Fernandez S, Pancheri JM, Sun $\mathrm{X}$, et al. Infliximab for intensification of primary therapy for Kawasaki disease: a phase 3 randomised, double-blind, placebocontrolled trial. Lancet. 2014; 383(9930): 1731-8. https://doi.org/10.1016/S0140-6736(13)62298-9.

[4] Nanishi E, Nishio H, Takada H, Yamamura K, Fukazawa M, Furuno $\mathrm{K}$, et al. Clarithromycin plus intravenous immunoglobulin therapy can reduce the relapse rate of Kawasaki disease: a phase 2, open-label, randomized control study. J Am Heart Assoc. 2017 Jul; 6(7): e005370. Published online 2017 Jul 6. doi: 10.1161/JAHA.116.005370.

[5] Hamada H, Suzuki H, Onouchi Y, Ebata R, Terai M, Fuse S, et al. Efficacy of primary treatment with immunoglobulin plus ciclosporin for prevention of coronary artery abnormalities in patients with Kawasaki disease predicted to be at increased risk of non-response to intravenous immunoglobulin (KAICA): a randomised controlled, open-label, blinded-endpoints, phase 3 trial. Lancet. 2019; 393(10176):1128-37. http://dx.doi.org/10.1016/S01406736(18)32003-8.

[6] Grignani R, Quek SC. Kawasaki disease in 2019-past controversies, present insights and future directions. Pediatr Med 2019; 2: 10. http://dx.doi.org/10.21037/pm.2019.03.03.

[7] McCrindle BW, Rowley AH, Newburger JW, Burns JC, Bolger AF, Gewitz MG, et al. Diagnosis, treatment, and long-term management of Kawasaki Disease: a scientific statement for health professionals from the American Heart Association. Circulation 2017; 135: e92799. DOI:10.1161/CIR.0000000000000484.

[8] McCrindle BW, Tierney ESS. Acute treatment for Kawasaki disease: challenges for current and future therapies. J Pediatr 2017; 184: 7-10. DOI: 10.1016/j.jpeds.2017.01.072.

[9] Kuo HC, Guo MM, Lo MH, Hsieh KS, Huang YH. Effectiveness of intravenous immunoglobulin alone and intravenous immunoglobulin combined with high-dose aspirin in the acute stage of Kawasaki 
disease: study protocol for a randomized controlled trial. BMC Pediatr. 2018; 18: 200. https://doi.org/10.1186/s12887-018-1180-1.

[10] Nakada T. Effects of anti-inflammatory drugs on intravenous immunoglobulin therapy in the acute phase of Kawasaki disease. Pediatr Cardiol 2015; 36: 335-9. DOI 10.1007/s00246-014-1010-7.

[11] Lau AC, Duong TT, Ito S, Yeung RS. Intravenous immunoglobulin and salicylate differentially modulate pathogenic processes leading to vascular damage in a model of Kawasaki disease. Arthritis Rheum 2009; 60: 2131-41. https://doi.org/10.1002/art.24660.

[12] Cho HJ, Bak SY, Kim SY, Yoo R, Baek HS, Yang S, et al. High neutrophil: lymphocyte ratio is associated with refractory Kawasaki disease. Pediatr Int 2017; 59: 669-74. https://doi.org/10.1111/ped.13240|.

[13] Nakada T. Acute phase treatment for prevention of coronary artery stenosis caused in Kawasaki disease: a single center retrospective study. Journal of Advanced Research in Medicine 2018; 5(4): 1-7. https://doi.org/10.24321/2349.7181.201818.

[14] Ayusawa M, Sonobe T, Uemura S, Ogawa S, Nakamura Y, Kiyosawa $\mathrm{N}$, et al. Revision of diagnostic guidelines for Kawasaki disease (the 5 th revised edition). Pediatr Int 2005; 47: 232-4. https://doi.org/10.1111/j.1442-200x.2005.02033.

[15] Ayusawa M. The diagnostic guidelines for Kawasaki disease (the 6th revised edition). Japanese Journal of Pediatrics 2020; 73 (2): 253-7.

[16] Rearch Committee of the Japanese Society of Pediatric Cardiology; Cardiac Surgery Committee for Development of Guidelines for Medical Treatment of Acute Kawasaki Disease. Guidelines for medical treatment of acute Kawasaki disease: report of the Research Committee of the Japanese Society of Pediatric Cardiology and Cardiac Surgery (2012 revised version). Pediatr Int 2014; 56: 135-58. https://doi.org/10.1111/ped.12317.

[17] Hirata S, Nakamura Y, Yanagawa H. Incidence rate of recurrent Kawasaki disease and related risk factors: from the results of nationwide surveys of Kawasaki disease in Japan. Acta Paediatr. 2001; $\quad 90(1): \quad 40-4 . \quad$ https://doi.org/10.1111/j.16512227.2001.tb00253.x

[18] Nakada T. Usefulness of an initial single intravenous immunoglobulin infusion with delayed use of aspirin against Kawasaki disease relapse: a single-center retrospective study. Journal of Advanced Research in $\begin{array}{lllll}\text { Medicine } & 2019 ; & 6 & \text { (1): }\end{array}$ https://doi.org/10.24321/2349.7181.201905.

[19] Nakada T. Risk stratification after initial therapy for intravenous immunoglobulin-resistant Kawasaki disease. Research Journal of Life Sciences, Bioinformatics, Pharmaceutical and Chemical Sciences 2019; 5 (5): 1-13. DOI: 10.26479/2019.0505.01.

[20] Kobayashi T, Inoue Y, Takeuchi K, OkadaY, Tamura K, Tomomasa $\mathrm{T}$, et al. Prediction of intravenous immunoglobulin unresponsiveness in patients with Kawasaki disease. Circulation 2006; 113:2606-12. http://www.circulationaha.org DOI:10.1161/CIRCULATIONAHA.105.592865

[21] Nakada T. Intravenous immunoglobulin therapy for prevention of coronary artery stenosis caused in Kawasaki disease. Internationa Journal of Advances in Medical Sciences 2018; 3 (6): 1-13 www.kibanresearchpublications.com.

[22] Fukazawa R, Kobayashi T, Mikami M, Saji T, Hamaoka K, Kato H, et al. Nationwide survey of patients with giant coronary aneurysm secondary to Kawasaki disease 1999-2010 in Japan. Circ J 2018; 82 239-46. https://doi:10.1253/circj.CJ-17-0433.

[23] Tsuda E, Tsujii N, Hayama Y. Stenotic lesions and the maximum diameter of coronary artery aneurysm in Kawasaki disease. J Pediatr 2019; 194: 165-70. https://doi.org10.1016/j.jpeds.2017.09.077.

[24] Nakada T. Acute phase treatment for infants younger than 1 year of age with Kawasaki disease: a single center retrospective study. Journal of Advanced Research in Medicine 2019; 6 (1): 1-6. https://doi.org/10.24321/2349.7181.201901.

[25] Takahara T, Yamagami Y, Oonishi S, Ohba H, Suga T, Chujyo S. Therapeutic strategy for immunoglobulin refractory Kawasaki disease including plasma exchange therapy in 60 patients. Progress in Medicine 2014; 34: 1282-7.

[26] Sonoda K, Mori M, Hokosaki T, Yokota S. Infliximab plus plasma exchange rescue therapy in Kawasaki disease. J Pediatr 2014; 164 1128-32. https://doi.org/10.1016/j.jpeds.2014.01.020.

[27] Rigante D, Andreozzi L, Fastiggi M, Bracci B, Natale MF, Esposito $\mathrm{S}$, et al. Critical overview of the risk scoring systems to predict nonresponsiveness to intravenous immunoglobulin in Kawasaki syndrome. Int J Mol Sci; 2016. DOI: 10.3390/ijms17030278.

[28] Nakada T. Background factors associated with the complications of coronary artery lesions caused by Kawasaki disease. Clinical Medicine Research. 2015; 4: 127-31. Published online July 30, 2015 (http://www.sciencepublishinggroup.com $/ \mathrm{j} / \mathrm{cmr}$ ) 10.11648/j.cmr.20150405.11.
[29] Makino N, Nakamura Y, Yashiro M, Kosami K, Matsubara Y, Ae R, et al. Nationwide epidemiologic survey of Kawasaki disease in Japan, 2015-2016. Pediatr Int 2019; 61: 397-403. https://doi.org/10.1111/ped.13809.

[30] Nakada T. Varying relapse rates in Kawasaki disease among different initial therapies. International Journal of Pharmacy and Pharmaceutical Research. Human. 2019; 16 (3): 175-188. www.ijppr.humanjournals.com.

[31] Miyata K, Kaneko T, Morikawa Y, Sakakibara H, Matsushima T, Misawa M, et al. Efficacy and safety of intravenous immunoglobulin plus prednisolone therapy in patients with Kawasaki disease (Post RAISE): a multicentre, prospective cohort study. Lancet Child Adolesc Health. 2018; 2(12):855-862. https://doi.org/10.1016/S23524642(18)30293-1

[32] Fukui M, Nakamura T, Abe Y, Yagawa A, Yamasaki T, Mikawa T, et al. An infant with Kawasaki disease followed by femoral osteomyelitis. The Journal of the Japan Pediatric Society. 2016; 120 (7): 1094-8.

[33] Chbeir D, Gaschignard J, Bonnefoy R, Beyler C, Melki I, Faye A, et al. Kawasaki disease: abnormal initial echocardiogram is associated with resistance to IVIg and development of coronary artery lesions. Pediatr Rheumatol Online J. 2018; 16(1): 48. https://doi.org/10.1186/s12969-018-0264-7.

[34] Friedman KG, Gauvreau K, Hamaoka-Okamoto A, Tang A, Berry MS, Tremoulet A, et al. Coronary artery aneurysms in Kawasaki disease: risk factors for progressive disease and adverse cardiac event in the US population. J Am Heart Assoc. 2016; 5: e003289 https://doi:10.1161/JAHA.116.003289.

[35] Nakada T. Outcomes in patients with Kawasaki disease associated with coronary artery lesions before initial therapy. Med J Aomori 2020; 65 (1): 1-7.

[36] Algonaid OA, Almoukirish AS, Almashham YH. Giant right coronary aneurysm secondary to Kawasaki disease in an infant. J Saudi Heart Assoc 2019; 31: 9-11. https://doi.org/10.1016/j.jsha.2018.08.004.

[37] Ashida A, Ozaki N, Kishi K, Katayama H, Okasora K, Tamai H. A case report of refractory Kawasaki disease with bilateral giant coronary aneurysms treated with intravenous immunoglobulin and prednisolone combination therapy. Progress in Medicine 2013; 33: 1471-4

[38] Dionne A, Burns JC, Dahdah N, Tremoulet AH, Gauvreau K, de Ferranti SD, et al. Treatment intensification in patients with Kawasaki disease and coronary aneurysm at diagnosis. Pediatrics. 2019; 143(6). pii: e20183341. https://doi.org/10.1542/peds.2018-3341.

[39] Miura M, Kobayashi T, Igarashi T, Hamada H, Iwata N, Sasaki Y, et al. Real-world safety and effectiveness of infliximab in pediatric patients with acute Kawasaki disease: A postmarketing surveillance in Japan (SAKURA Study). Pediatr Infect Dis J 2020; 39 (1): 41-7. doi: 10.1097/INF.0000000000002503.

[40] Nakada T. Combined usefulness of serum albumin and C-reactive protein for guiding rescue therapy for intravenous immunoglobulinresistant Kawasaki disease. International Journal of Advances in Medical Sciences 2018; 3 (3): 1-7. www.kibanresearchpublications.com.

[41] Nakada T. Usefulness of the neutrophil to lymphocyte ratio for risk stratification after initial intravenous immunoglobulin therapy in Kawasaki disease. Research Journal of Life Sciences, Bioinformatics, Pharmaceutical and Chemical Sciences 2017; 2 (5): 1-17. www.rjlbpcs.com.

[42] Ha KS, Lee J, Jang GY, Lee J, Lee KC, Son CS, et al. Value of neutrophil-lymphocyte ratio in predicting outcomes in Kawasaki disease. Am J Cardiol 2015; 116: 301-6. http://dx.doi.org/10.1016/j.amjcard.2015.04.02.

[43] Nakamura Y, Yanagawa H, Ojima T, Kawasaki T, Kato H. Cardiac sequelae of Kawasaki disease among recurrent cases. Arch Dis Child 1998; 78: 163-5.

[44] Maddox RA, Holman RC, Uehara R, Callinan LS, Guest JL, Schonberger LB, et al. Recurrent Kawasaki disease: USA and Japan. Pediatr Int 2015; 57(6): 1116-1120. https://doi.org/10.1111/ped.12733.

[45] Nakada T. Clinical features of patients with recurrent Kawasak disease. Nippon Rinsho 2008; 66 (2): 296-300. PMID: 18260328.

[46] Aggarwal V, Sexson-Tejtal K, Lam W, Valdes SO, dela Uz CM, Kim $\mathrm{JJ}$, et al. The incidence of arrhythmias during exercise stress tests among children with Kawasaki disease: A single-center case series. Congenit Heart Dis 2019; 14 (6): 1032-6. https://doi.org/10.1111/chd.12864.

[47] Nakada T. Ventricular arrhythmia and possible myocardial ischemia in late stage Kawasaki disease: patient with a normal coronary arteriogram. Acta Paediatrica Japonica 1996; 38: 365-9. https://doi.org/10.1111/j.1442-200X.1996.tb03508. 


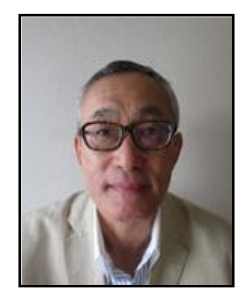

Dr Toshimasa Nakada

Birth of place: Japan. Birth of date: 20/02/1957.

I received medical education at Hirosaki

University School of Medicine. I had MD

regarding pediatrics. I have been working as a pediatrician at Department of Pediatrics, Aomori

Prefectural Central Hospital. Aomori City, Aomori

Prefecture, Japan, from 1988 to the present time.

My major field of study is general pediatric cardiology: Kawasaki disease, arrhythmia, congenital heart disease, cardiomyopathy, heart disease screening, and preventive medicine. Acute phase treatment for Kawasaki disease using an initial single intravenous immunoglobulin therapy is one of my lifeworks. The recent publications are listed in this article.

The most recent publication is the article entitled "Preventive therapies using sodium channel blockers for congenital long QT syndrome" [World Journal of Pharmaceutical and Life Sciences 2020; 6 (6): 7-12]. 